\title{
Extended Hückel theory for band structure, chemistry, and transport. II. Silicon
}

\author{
D. Kienle, ${ }^{\text {a) }}$ K. H. Bevan, G.-C. Liang, and L. Siddiqui \\ Department of Electrical and Computer Engineering, Purdue University, West Lafayette, Indiana 47907
}

\section{J. I. Cerda}

Instituto de Ciencia de Materiales de Madrid, CSIC, Cantoblanco, 28049 Madrid, Spain

\author{
A. W. Ghosh \\ Department of Electrical and Computer Engineering, University of Virginia, Charlottesville, Virginia 22903
}

(Received 11 April 2006; accepted 5 July 2006; published online 29 August 2006)

\begin{abstract}
In this second paper, we develop transferable semiempirical extended Hückel theoretical (EHT) parameters for the electronic structure of another technologically important material, namely, silicon. The EHT parameters are optimized to experimental target values of the band dispersion of bulk silicon. We quantitatively benchmark our parameters to bulk electronic properties such as band edge energies and locations, effective masses, and spin-orbit coupling parameters, competitive with a nearest-neighbor $s p^{3} d^{5} s^{*}$ orthogonal tight-binding model for silicon of T. Boykin et al. [Phys. Rev. B 69, 115201 (2004)] that has been widely used to model silicon-based devices (see, e.g., A. Rahman et al. [Jpn. J. Appl. Phys. Part I 44, 2187 (2005)] and J. Wang et al. [Appl. Phys. Lett. 86, $093113(2005)])$. The transferability of the parameters is checked for multiple physical and chemical configurations, specifically, two different reconstructed surfaces, $\mathrm{Si}(100)-(2 \times 1)$ and $\mathrm{Si}(111)-(2 \times 1)$. The robustness of the parameters to different environments is demonstrated by comparing the surface band structures with density functional theory GW calculations and photoemission/inverse photoemission experiments. We further apply the approach to calculate the one-dimensional band dispersion of an unrelaxed rectangular silicon nanowire and explore the chemistry of surface passivation by hydrogen. Our EHT parameters thus provide a quantitative model of bulk silicon and silicon-based interfaces such as contacts and reconstructed surfaces, which are essential ingredients towards a quantitative quantum transport simulation through silicon-based heterostructures. (C) 2006 American Institute of Physics. [DOI: 10.1063/1.2259820]
\end{abstract}

\section{INTRODUCTION}

Silicon constitutes the dominating component of present day microelectronic devices. The extended infrastructure of the chip industry and the well studied material and processing properties of silicon argue in favor of retaining it as the element of choice for future nanoscale devices, possibly with modifications due to strain engineering, ${ }^{1-4}$ germanium alloying, 5,6 or hybrid "add-on" components such as organic thin films ${ }^{7-9}$ or molecular assemblies. ${ }^{10,11}$ It is now becoming quite clear that the increasing importance of surface scattering in nanoelectronic devices requires a reevaluation of standard electronic structure techniques, typically calibrated to bulk band structure but not to surface chemical or transport properties.

Numerous recent experiments highlight the importance of the detailed interaction between bulk band structure and surface chemical properties of silicon. These include gating of transistors by surface dimers, ${ }^{12,13}$ detection of single molecule adsorbates by silicon substrates, ${ }^{14,15}$ systematic variation in molecular spectra that can be correlated with bonding variations, ${ }^{16}$ and predictable negative differential resistance (NDR), possibly due to the interaction between molecular

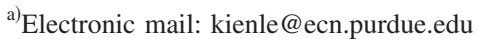

levels and the silicon band edge (Refs. 17-19). ${ }^{1}$ It seems that future devices might require an understanding and perhaps even a precision engineering of interfacial properties with silicon. Thus even a minimal quantitative model of transport through silicon devices must be able to capture its multiple band edge energies and locations, effective masses, spinorbit couplings, band-bending and depletion electrostatics, surface reconstruction, and associated defect state electronic properties.

Density functional theoretical (DFT) approaches, such as local density approximation (LDA) and generalized gradient approximation (GGA), are well suited to determining electronic and material properties such as molecular configuration which depend on the total energy. ${ }^{21-23}$ They are, however, questionable for transport, ${ }^{24,25}$ which depends instead on the accuracy of a few levels or bands near the Fermi energy. Even the band gap of semiconductors, a crucial ingredient for modeling semiconductor electronic transport, is underestimated by DFT-LDA/GGA calculations. ${ }^{26}$ While the band gap itself can be fixed within the GW approxi-

${ }^{1}$ Although the observed NDR has features that qualitatively agree with the
proposed mechanism, specifically, a reversal of polarity with doping and a
migration of the onset voltage with tip withdrawal, there are issues to be
sorted out, such as the accessibility of the levels ${ }^{17-20}$ and the possibility that
these NDR events are driven by scattering-induced configuration changes. ${ }^{20}$ 
mation, ${ }^{27,28}$ these methods are computationally expensive and currently not very practical for transport simulations through nano interfaces, besides the need to benchmark with other important band structure parameters.

In less rigorous, but computationally cheaper semiempirical approaches, the band structure is determined by adjusting irreducible matrix elements of the Hamiltonian and overlap matrices to match band edge positions and effective masses. While, orthogonal tight-binding (OTB) parameters have been extensively optimized for electronic structure of bulk semiconductors such as silicon and germanium, ${ }^{29-31}$ their transferability to various reconstructed surfaces has not been quantitatively benchmarked, ${ }^{32,33}$ so that usually a reparametrization is required. Another shortcoming is the lack of explicit basis sets to capture electronic density variations on an interatomic scale where bonding chemistry sets in. On the other hand, orthogonal as well as non-orthogonal tightbinding parametrization schemes have been proven to be quite robust for total energy calculations to determine the equilibrium atomic structure of (reconstructed) silicon surfaces with different orientations ${ }^{34-42}$ as well as the atomic structure of finite sized silicon clusters. ${ }^{43-51}$ Since the total energy is an integral rather than a spectral property, the atomic structure is less sensitive with respect to variations in the electronic spectrum of the system, since potential errors partly cancel out. ${ }^{52}$ The main difficulty here is to construct an accurate functional for the total energy, so that atomic structures, electronic affinities, etc., can be predicted in a quantitative manner. $^{51}$

In our preceding paper, we used a nonorthogonal tightbinding scheme based on extended Hückel theory (EHT) to model carbon nanotubes, demonstrating the transferability of the EHT parameters from a graphene sheet to small diameter tubes, and thereafter applying to strongly deformed carbon nanotube (CNT) molecule heterostructures. ${ }^{53}$ Here, we present optimized EHT parameters for bulk silicon that will be benchmarked against multiple target values such as band edges and effective masses. We then explore the transferability of these EHT parameters to different environments by calculating the two-dimensional (2D) band structure for two reconstructed silicon surfaces, silicon $(100)-(2 \times 1)$ and (111) $-(2 \times 1)$, and compare them quantitatively to experiments and state of the art DFT-GW calculations. Finally, we use the silicon parameters to calculate the one-dimensional (1D) band dispersion and transmission for an unreconstructed silicon nanowire to examine its surface passivation.

We have used this approach in the past to successfully deconstruct the role of silicon surface microstructure on the electronic properties of buckyballs. ${ }^{16}$ It was also used to develop a quantitative model of molecular NDR on silicon, ${ }^{19}$ and to examine the role of surface relaxation on silicon nanowire transistor currents. ${ }^{54}$ Our approach thus achieves a good match between computational accuracy and practicality, while its explicit basis sets allow us to formulate quantum scattering boundary conditions that can help us combine multiscale atomic and coarse grain descriptions of different parts of a nanostructure. ${ }^{55}$

The paper is organized as follows: Sec. II briefly summarizes the main features of extended Hückel theory. Section
III discusses the optimized EHT parameters for bulk silicon and the comparison of the band edges and effective masses to experimental target values. We then investigate the transferability of the parameters by employing them to different silicon surfaces. Finally, the 1D electronic structure of a silicon nanowire is determined including its surface passivation. We summarize present and future work in Sec. IV.

\section{APPROACH}

The silicon band structures for the bulk, the two reconstructed surfaces, and the 1D nanowire are calculated within a nonorthogonal Slater-Koster scheme ${ }^{56}$ using extended Hückel theory to generate the overlap- and Hamiltonianmatrix elements $\mathbf{S}$ and $\mathbf{H}$, respectively. Here, we briefly summarize the essential features of EHT, which is described in more detail in Refs. 53 and 57.

The most striking difference between EHT and OTB is that in EHT one works with explicit atomiclike orbital (AO) basis functions, which are used to construct the matrix elements $\mathbf{S}$ and $\mathbf{H}$. In turn, in orthogonal tight binding the basis functions are not known and used as a formal tool to construct all matrix elements of the Hamiltonian. The matrix elements are then usually adjusted to a reference band structure, for example. Compared to OTB in extended Hückel theory one adjusts only the diagonal matrix elements of the Hamiltonian (onsite energies $E_{\mu \mu}$ ) and the parameters specifying the basis functions, which are Slater-type functions (STO) ${ }^{53,58}$ Since the basis functions are known, the overlap matrix $\mathbf{S}$ is calculated explicitly and used to construct the off-diagonal matrix elements of the Hamiltonian (hopping) according to ${ }^{53,57}$

$$
\begin{aligned}
& H_{\mu \mu}=E_{\mu \mu}, \\
& S_{\mu \nu}=\int d^{3} \mathbf{r} \phi_{\mu}^{*}(\mathbf{r}) \phi_{\nu}(\mathbf{r}), \\
& H_{\mu \nu}=\frac{1}{2} K_{\mathrm{EHT}} S_{\mu \nu}\left(H_{\mu \mu}+H_{\nu \nu}\right),
\end{aligned}
$$

assuming that the Hamiltonian depends linearly on the overlap. ${ }^{57}$ The equations can be generalized to model heterogeneous structures and interfaces. ${ }^{53,59,60} S_{\mu \nu}$ is the overlap matrix between the orbital basis functions $\phi_{\mu}$ and $\phi_{\nu}$, while $K_{\mathrm{EHT}}$ is an additional fitting parameter usually set to 1.75 for molecules and 2.3 for solids. ${ }^{57,58}$

Compared to OTB, Slater-type basis functions are nonorthogonal, providing an improved transferability of the model parameters with respect to changes in the environment. ${ }^{23,61,62}$ The enhanced transferability can be justified by constructing orthogonal Löwdin orbitals from the nonorthogonal basis functions. Compared to the original AOs these Löwdin functions are longer ranged to enforce orthogonality over the entire domain, making the basis functions more sensitive to environmental changes. ${ }^{52,56,61,63}$

The transferability of parameters becomes particularly evident under structural deformation. For bulk systems empirical scaling rules have been developed to handle small amounts of strain within nearest-neighbor orthogonal tight binding, ${ }^{64,65}$ but their transferability becomes questionable 
for reconstructed surfaces where structural deformations are often much greater than 2\%-5\%, along with associated changes in bonding chemistry. The scaling laws are often of the power-law type with an adjusted exponent to match elastic properties of bulk systems valid for small strain, and may thus be inapplicable to surfaces. Furthermore, the surface band structures are critically dependent on interdimer interactions, which are hard to capture within a nearest-neighbor approximation.

\section{RESULTS FOR EHT-ELECTRONIC STRUCTURE FOR SILICON}

\section{A. Si Bulk}

To perform quantitative transport calculations through nanostructure materials, the free parameters of a semiempirical tight-binding model have to be calibrated to experimental targets and/or band structure data obtained from other theoretical approaches. In the past, EHT parameters were generated for several bulk crystal structures such as metals, semi-conductors, and compounds. ${ }^{58}$ Specifically, for silicon these parameters have been optimized to match the bulk dispersion of DFT-GW calculations of Rohlfing et al. ${ }^{28}$ at selected points within the three dimensional (3D) Brillouin zone. $^{58}$

We generate a more elaborate parametrization using experimentally determined band structure values of bulk silicon, such as band edge locations and effective masses as targets. ${ }^{66}$ We use the TBGREEN code ${ }^{67}$ to minimize the rootmean-square (rms) error between our EHT bands and the targets via a conjugate gradient method as described in Ref. 58. Since most of the targets refer to experiments done at low temperatures [5-10 K (Ref. 66)], we perform the minimization of the rms error at $T=0.0 \mathrm{~K}$. The 3D band structure of silicon is calculated using an $s p^{3} d^{5}$ orbital basis for each silicon atom. In order to capture and optimize the split-off gap $\Delta_{0}$, the Hamiltonian is made spin dependent by incorporating spin-orbit $(\mathrm{SO})$ interaction. For simplicity we assume that the $\mathrm{SO}$ interaction $H^{\mathrm{SO}}$ is local, i.e., only spins on the same site interact. In this case, the respective SO Hamiltonian is given by ${ }^{68}$

$$
H^{\mathrm{SO}}=\sum_{\mu l} \chi_{\mu l} \sum_{m m^{\prime} \sigma \sigma^{\prime}}\left\langle\mu l m \sigma|\mathbf{L} \cdot \mathbf{S}| \mu l m^{\prime} \sigma^{\prime}\right\rangle,
$$

where $|\mu l m \sigma\rangle$ denotes the $(l, m)$ AO basis function of atom $\mu$ in the unit cell with spin $\sigma$ and $\chi_{\mu l}$ is the spin-orbit parameter $(\mathbf{L} \cdot \mathbf{S}$ prefactor). Explicit expressions for the $\mathbf{L} \cdot \mathbf{S}$ matrix elements may be found in Ref. 68. In our calculations, we added the $H^{\mathrm{SO}}$ interaction only for the $p$ states $(l=1)$, thus introducing a further fit parameter, the $L S$ prefactor, which we denote by $L S_{p}$.

Figure 1 shows the band dispersion for bulk silicon using the optimized silicon atom EHT parameters given in Table I. Table II shows the results of our optimization along with their relative errors (columns 2 and 3) compared with the experimental target values as reference (column 4). Our values for the band edges and the effective masses agree very well with the target values, ${ }^{66}$ and are competitive with state-

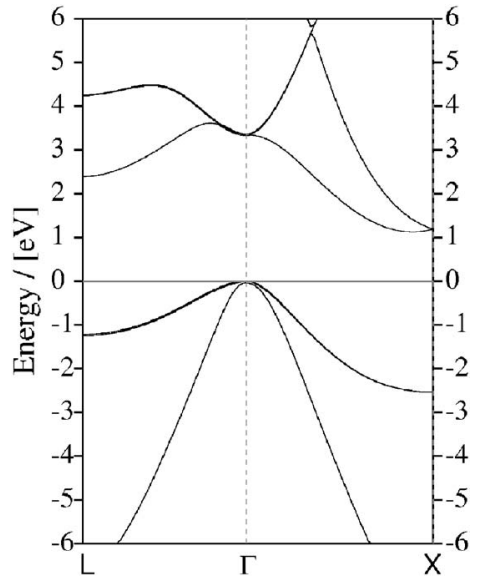

FIG. 1. Band structure of bulk silicon calculated within EHT using the parameters given in Table I. The Fermi level is at $E_{F}=0.0 \mathrm{eV}$ (horizontal solid line). The EHT parameters are optimized to experimental target values taken from Ref. 66.

of-the-art orthogonal $s p^{3} d^{5} s^{*}$ tight-binding model for bulk silicon ${ }^{31}$ whose results and relative errors are shown in columns 5 and 6 , respectively.

\section{B. Si Surfaces for different orientations}

We now investigate the transferability of our EHT parameters for bulk silicon and use them to calculate non-selfconsistent surface band structures for two different surface orientations. The semi-infinite surfaces are modeled by a finite slab consisting of a series of layers, with the dangling bond states at the bottom eliminated by hydrogen passivation.

To calculate the 2D silicon band structure of the reconstructed surface we use the unit cell coordinate of Ref. 69 for $\mathrm{Si}(100)-(2 \times 1)$ and for $\mathrm{Si}(111)-(2 \times 1)$ from Ref. 70 as shown in Fig. 2. Since the unit cell of the more common $(7 \times 7)$ reconstruction for $\mathrm{Si}(111)$ is very large, we opted instead for the less common $(2 \times 1)$ reconstruction on this surface. For $\operatorname{Si}(100)-(2 \times 1)$ we use a slab with 13 layers, where the first four layers correspond to the reconstructed silicon surface, and the remaining eight layers correspond to positions of bulk silicon. The last layer consists of hydrogen to passivate the bottom of the slab. The lattice vectors for $\operatorname{Si}(100)-(2 \times 1)$ are $\mathbf{a}_{1}=7.68 \AA \mathbf{e}_{x}$ and $\mathbf{a}_{2}=3.84 \AA \mathbf{e}_{y}$. Similarly, the $\mathrm{Si}(111)-(2 \times 1)$ reconstructed surface contains 21 layers, where the first 8 layers are relaxed, the following 12

TABLE I. EHT parameters for bulk silicon using $s p d$ orbitals after optimization against the experimental target values specified in Table II. $E_{\text {on }}$ is the onsite energy, $\left\{\zeta_{i}, c_{i}\right\}$ are the exponents and respective expansion coefficients of the STO, and $L S_{p}$ denotes the parameter for spin-orbit coupling. The parameter $K_{\mathrm{EHT}}$ is taken as orbital independent.

\begin{tabular}{lcccccccc}
\hline \hline & $\mathrm{AO}$ & $E_{\text {on }}$ & $\zeta_{1}$ & $c_{1}$ & $\zeta_{2}$ & $c_{2}$ & \multicolumn{1}{c}{$L S_{p}$} & $K_{\text {EHT }}$ \\
\hline $\mathrm{Si}$ & $3 s$ & -17.489 & 2.170 & 1.0 & 0.0 & 0.0 & $\cdots$ & 2.622 \\
& $3 p$ & -10.535 & 1.853 & 1.0 & 0.0 & 0.0 & 0.044 & 2.622 \\
& $3 d$ & -4.911 & 0.880 & 0.759 & 0.0 & 0.0 & $\cdots$ & 2.622 \\
\hline \hline
\end{tabular}


TABLE II. Band structure characteristics for bulk silicon using extended Hückel theory and spd orbitals for each Si atom. The EHT parameters, cf. Table I, have been optimized to experimental target values taken from Ref. 66 (column 4). Columns 5 and 6 contain the fit values and errors based on an orthogonal tight-binding model using $s p^{3} d^{5} s^{*}$ orbitals (see Ref. 31). The effective masses at the $L$ valley are not well established, so that we do not strongly weigh them in the optimization (see Ref. 31).

\begin{tabular}{cccccc}
\hline \hline Quantity & Si-EHT & Rel. err. $(\%)$ & Si-target & Si-sp $p^{3} d^{5} s^{*}$ & Rel. err. $(\%)$ \\
\hline$E_{c}^{\Gamma}$ & 3.324 & 1.3 & 3.368 & 3.999 & 0.9 \\
$E_{v}^{\Gamma}$ & 0.0 & 0.0 & 0.0 & 0.0 & 0.0 \\
$\Delta_{0}^{\Gamma}$ & 0.0445 & 1.0 & 0.045 & 0.0472 & 4.9 \\
$E_{c, \min }^{L}$ & 2.393 & 0.3 & 2.400 & 2.383 & 0.7 \\
$E_{c, \min }^{X}$ & 1.122 & -0.4 & 1.118 & 1.131 & 1.2 \\
$k_{\min }^{[001]}$ & $88.0 \%$ & -3.5 & $85.0 \%$ & $81.3 \%$ & 4.4 \\
$m_{X, l}^{(e)}$ & 0.939 & -2.5 & 0.916 & 0.891 & 2.7 \\
$m_{X, t}^{(e)}$ & 0.161 & 15.5 & 0.190 & 0.201 & 5.8 \\
$m_{L, l}^{(e)}$ & 1.136 & 43.2 & 2.000 & 3.433 & 71.7 \\
$m_{L, t}^{(e)}$ & 0.140 & -39.7 & 0.100 & 0.174 & 74.0 \\
$m_{l h}^{[001]}$ & -0.182 & 10.7 & -0.204 & -0.214 & 4.9 \\
$m_{l h}^{[110]}$ & -0.148 & -0.7 & -0.147 & -0.152 & 3.4 \\
$m_{l h}^{[111]}$ & -0.149 & -7.0 & -0.139 & -0.144 & 3.6 \\
$m_{h h}^{[001]}$ & -0.277 & -0.9 & -0.275 & -0.276 & 0.4 \\
$m_{h h}^{[110]}$ & -0.579 & 0.0 & -0.579 & -0.581 & 0.3 \\
$m_{h h}^{[111]}$ & -0.663 & 10.2 & -0.738 & -0.734 & 0.5 \\
$m_{s o}$ & -0.217 & 7.1 & -0.234 & -0.246 & 5.1 \\
$E_{1}^{G}$ & -12.11 & 3.1 & -12.50 & $\ldots$ & $\ldots$ \\
$m_{1}^{G}$ & 1.77 & -47.7 & 1.20 & $\ldots$ & $\ldots$ \\
\hline \hline
\end{tabular}

layers have a bulk silicon geometry, while the last layer is hydrogen. The $2 \mathrm{D}$ Bravais lattice vectors here are $\mathbf{a}_{1}=6.65 \AA \mathbf{e}_{x}$ and $\mathbf{a}_{2}=3.84 \AA \mathbf{e}_{y}$.

Figure 3 shows the band structure of reconstructed silicon $(100)-(2 \times 1)$ calculated within EHT (top) using the silicon parameters of Table I. The band dispersion at the bottom of Fig. 3 corresponds to DFT-GW calculations of Rohlfing et $a .^{71}$ with the $\pi$ and $\pi^{*}$ bands shown in solid lines. The diamond symbols represent photoemission (PES) experiments. As can be seen, the shapes of our EHT-calculated $\pi$

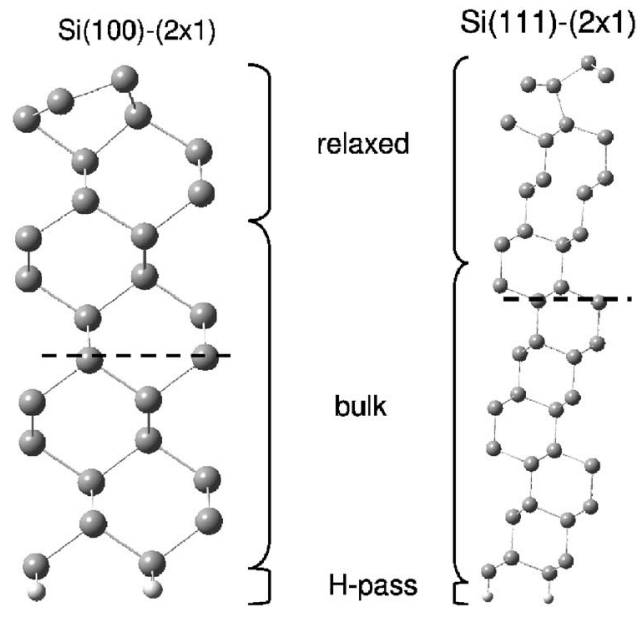

FIG. 2. Structure of the unit cell for the two silicon surfaces (see Refs. 69 and 70). Left: $\operatorname{Si}(100)-(2 \times 1)$ with the first four layers relaxed and nine bulklike layers. Right: $\operatorname{Si}(111)-(2 \times 1)$ where the first 8 layers are relaxed and 12 bulk layers. In each case, the bottom of the surface is hydrogen passivated to remove dangling bond states. The dashed horizontal line indicates the position of one layer.
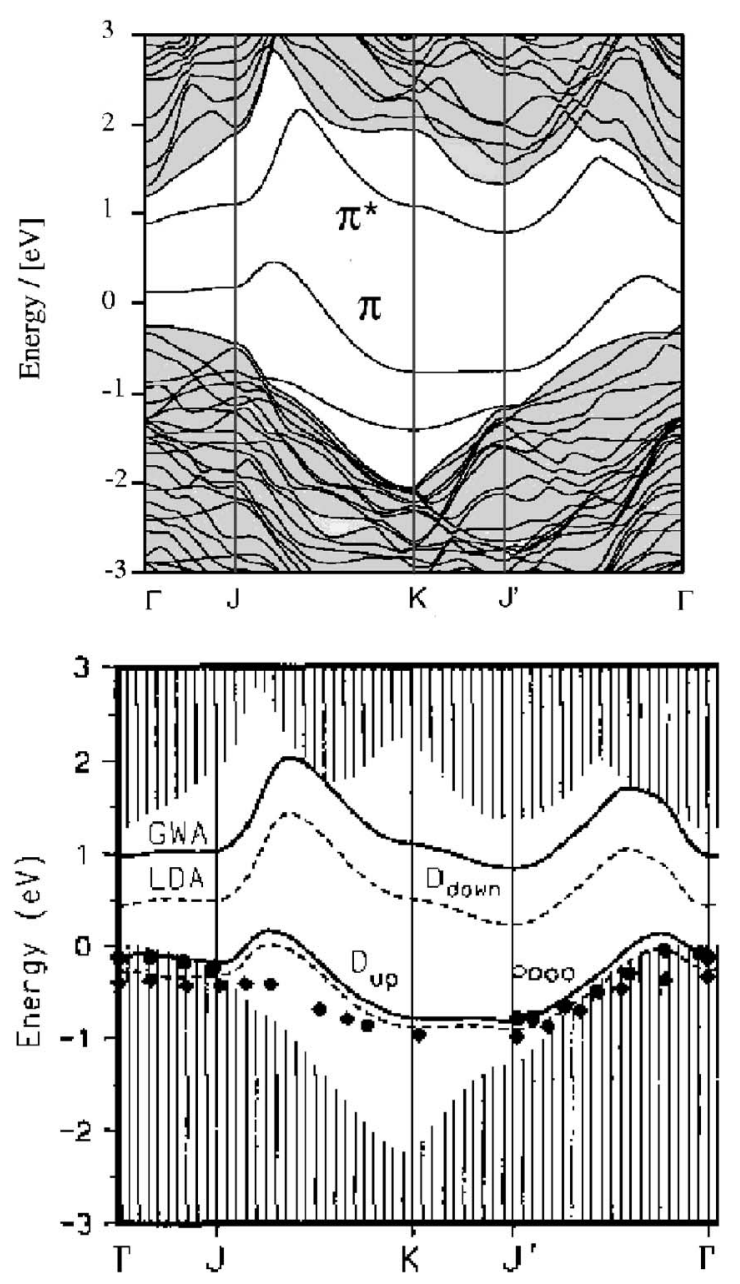

FIG. 3. Surface band structure of unpassivated, reconstructed silicon (100)- $(2 \times 1)$ calculated within EHT (top) using the parameters of Table I. The bottom figure is a DFT-GW calculation (see Ref. 71). The two bands within the 2D-projected bulk silicon band gap correspond to the $\pi$ and $\pi^{*}$ bands of the silicon surface described by the asymmetric dimer model. Reprinted figure (bottom) with permission from Ref. 28.

and $\pi^{*}$ surface bands agree very well qualitatively with DFT-GW calculations as well as with PES experiments for the $\pi$ band.

Despite the good qualitative agreement, which demonstrates the transferability of our parameters in capturing the essential physics of the surface bands, there are quantitative differences. The experimental indirect band gap $\Delta_{\pi^{*}-\pi}$ between the $\pi^{*}$ and $\pi$ band is about $\sim 0.8-1.2 \mathrm{eV},{ }^{72}$ whereas the gap in EHT, $\sim 0.3 \mathrm{eV}$, is underestimated as in DFT-LDA calculations, because we are overestimating the bandwidth of our $\pi$ bands. In Table III we compare the band gaps and band edges calculated in EHT at different points of the 2D Brillouin zone with those obtained by DFT-GW calculations $^{71}$ and experiments.

The difference between the theoretical approaches becomes more explicit in Fig. 4 where only the dispersions for the $\pi$ and $\pi^{*}$ surface bands are shown for our EHT calculation and the DFT-GW calculations of Rohlfing et al. ${ }^{71}$ The plots have been extracted from Fig. 3 by digitizing the respective $\pi$ and $\pi^{*}$ bands. The unoccupied $\pi^{*}$ band agrees quantitatively with the one obtained from DFT-GW over the entire Brillouin zone region as shown in Fig. 4. Similarly, the 
TABLE III. Comparison of the band gaps $\Delta$ and band edges $E$ calculated in EHT, DFT-GW (see Ref. 71), and experiments for the silicon surface $(100)-(2 \times 1)$ at different points of the $2 \mathrm{D}$ Brillouin zone. All values are in units of $\mathrm{eV}$. All experimental values have been extracted from the respective figure in Ref. 71 if not labeled otherwise.

\begin{tabular}{cccc}
\hline \hline & EHT (Table I) & DFT-GW & Expt. \\
\hline$E_{\max }^{\pi}$ & 0.47 & 0.16 & -0.42 \\
$E_{\min }^{\pi^{*}}$ & 0.81 & 0.81 & $\ldots$ \\
$\Delta_{\pi^{*}-\pi}$ & 0.34 & 0.65 & $\approx 0.8-1.2^{\mathrm{a}}$ \\
$E_{\Gamma}^{\pi}$ & 0.14 & -0.10 & $-0.13,-0.42$ \\
$E_{\Gamma}^{\pi^{*}}$ & 0.91 & 0.94 & $\ldots$ \\
$\Delta_{\Gamma}$ & 0.78 & 1.04 & $\ldots$ \\
$E_{J^{*}}^{\pi}$ & -0.20 & -0.19 & $-0.26,-0.26$ \\
$E_{J}^{\pi^{*}}$ & 1.11 & 1.00 & $\ldots$ \\
$\Delta_{J}$ & 0.91 & 1.19 & $\ldots$ \\
$E_{K}^{\pi}$ & -0.74 & -0.81 & -0.97 \\
$E_{K}^{\pi^{*}}$ & 1.11 & 1.07 & $\ldots$ \\
$\Delta_{K}$ & 1.85 & 1.87 & $\ldots$ \\
$E_{J^{\prime}}^{\pi}$ & -0.74 & -0.81 & $-0.81,-0.97$ \\
$E_{J^{\prime}}^{\pi^{*}}$ & 0.81 & 0.81 & $\ldots$ \\
$\Delta_{J^{\prime}}$ & 1.55 & 1.61 & $\ldots$ \\
\hline \hline
\end{tabular}

${ }^{\mathrm{a}}$ Reference 72.

$\pi$ band matches quantitatively DFT-GW as well over a wide range of the Brillouin zone, except within the $\Gamma-J$ and the first third of the $J-K$ path. The latter domain includes the $\pi$ maximum, which appears $\sim 0.25 / 0.3 \mathrm{eV}$ too high, so that the $\Delta_{\pi^{*}-\pi}$ band gap is noticeably underestimated.

The surface density of states (DOS) is shown in Fig. 5. The energy-resolved partial DOS is calculated for each dimer atom (upper and lower) and for the two deeper silicon layers away from the surface. The partial DOS of the upper dimer atom is located closer to the valence band, whereas for the lower one it lies near the conduction band, indicating that the $\pi$ surface band is formed from the upper dimer atom, whereas the $\pi^{*}$ band comes from the lower one. ${ }^{73}$ Consistent with the EHT $\pi$ band dispersions in Figs. 3 and 4 the PDOS of the upper dimer atom is too much above the valence band, so that the $\pi^{*}-\pi$ gap in the PDOS is too small. As we proceed away from the surface into the bulklike region, the weights of the $\pi$ and $\pi^{*}$ DOS decrease continuously (layer

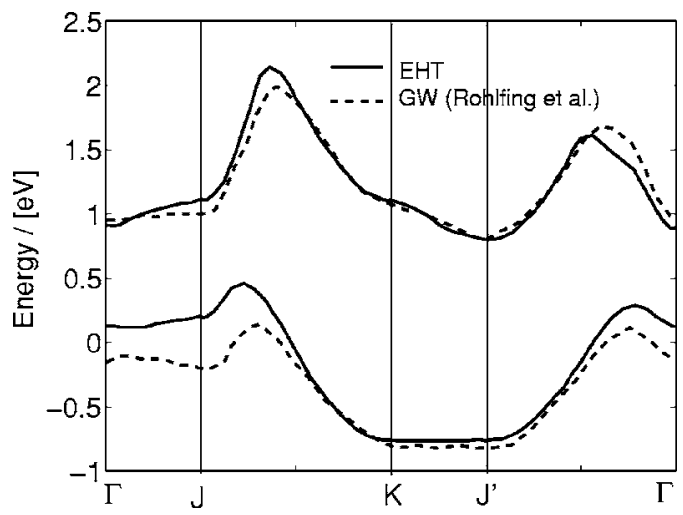

FIG. 4. Comparison of the $\pi$ and $\pi^{*}$ surface bands calculated within EHT and DFT-GW (see Ref. 71). The dispersion of the $\pi$ and $\pi^{*}$ bands of Fig. 3 have been digitized. The data of the red curve are adapted with permission from Fig. 3 of Ref. 28.

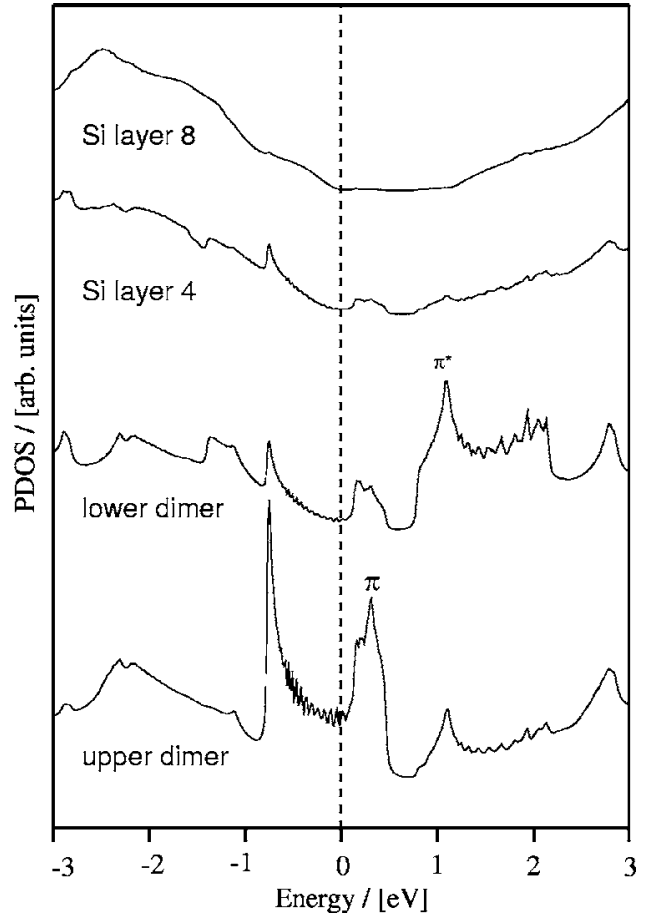

FIG. 5. Partial density of states (PDOS) of unpassivated silicon (100)-(2 $\times 1)$ surface calculated for different layers starting from the bulklike eighth layer (top) towards the (100) surface consisting of the two dimer atoms. The two peaks in the DOS at each dimer atom correspond to the $\pi$ and $\pi^{*}$ bands.

4), and completely disappear once a deeper bulklike layer is reached (layer 8), so that the original bulk band gap of $\sim 1.1 \mathrm{eV}$ is recovered.

As a second example, we look at the surface band structure of reconstructed $\mathrm{Si}(111)-(2 \times 1)$ as shown in Fig. 6 using the EHT parameters in Table I. Similar to the previous case, the overall shapes of the $\pi$ and $\pi^{*}$ surface bands match qualitatively with DFT-GW calculations of Rohlfing et al.
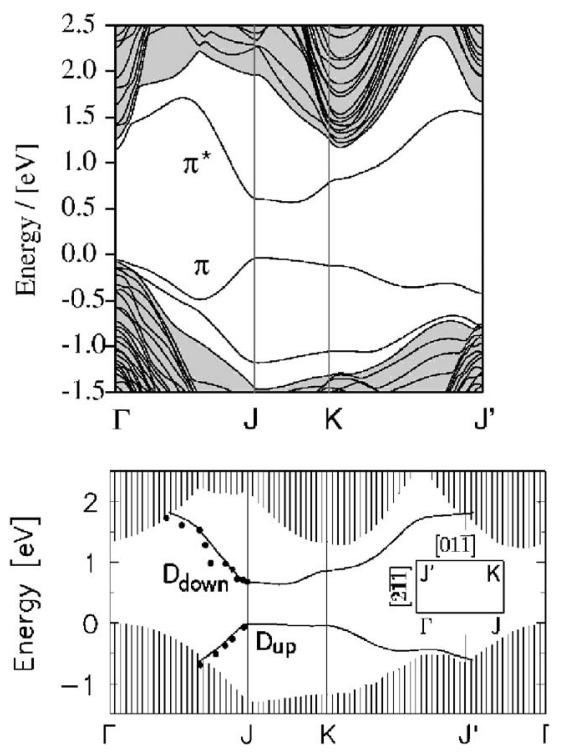

FIG. 6. $\pi$ and $\pi^{*}$ surface band structure of the unpassivated, reconstructed silicon (111)- $(2 \times 1)$ calculated in EHT (top). The figure at the bottom shows the dispersion calculated within DFT-GGA of Rohlfing and Louie (see Ref. 74), respectively. The bottom figure is reprinted with permission from Ref. 74. 
TABLE IV. Comparison of the band gaps $\Delta$ and bandedges $E$ (in units of eV) calculated in EHT, DFT-GW (see Refs. 74 and 75) and experiments for the silicon surface $(111)-(2 \times 1)$ at different points of the $2 \mathrm{D}$ Brillouin zone. Column 3 contains the values from Ref. 75 and column 4 the ones from Ref. 74. The experimental values (column 5) have been extracted from the corresponding figure in Ref. 74, respectively Ref. 75 if not labeled otherwise.

\begin{tabular}{lcccc}
\hline \hline & EHT (Table I) & DFT-GW & DFT-GW & Expt. \\
\hline$E_{J}^{\pi}$ & -0.05 & -0.07 & 0.0 & 0.09 \\
$E_{J}^{\pi^{*}}$ & 0.61 & 0.53 & 0.69 & 0.67 \\
$\Delta_{J}$ & 0.66 & 0.60 & 0.68 & 0.58 \\
$E_{K^{*}}^{\pi}$ & -0.11 & -0.04 & -0.04 & $\cdots$ \\
$E_{K}^{\pi^{*}}$ & 0.80 & 0.78 & 0.88 & $\ldots$ \\
$\Delta_{K}$ & 0.91 & 0.82 & 0.92 & $\ldots$ \\
\hline \hline
\end{tabular}

(Fig. 6 bottom).$^{74}$ In Table IV we compare our EHT band edges and gaps (column 1) at two specific points, $J$ and $K$, of the 2D Brillouin zone with DFT-GW calculations ${ }^{74,75}$ and PES/IPES experiments. The values for the band edges as well as for the gaps agree quantitatively among all three calculations, and also show a good agreement with PES/ inverse photoemission (IPES) experiments, where the error in the energy resolution is typically $150-200 \mathrm{meV}$ depending on temperature and incident energy of the electrons. A more extended comparison with PES/IPES experiments turns out to be very limited, since the $\pi$ and the $\pi^{*}$ bands are not as well experimentally determined as in the case of silicon (100)- $(2 \times 1)$.

Contrary to the previous case of $\mathrm{Si}(100)-(2 \times 1)$, we find for $\operatorname{Si}(111)-(2 \times 1)$ that both $\pi^{*}$ and $\pi$ band dispersions calculated in EHT agree very well quantitatively with DFT-GW calculations of Northrup et $a l^{75}$ and in particular with Rohlfing et $a l .{ }^{74}$ over the entire range of the Brillouin zone, as shown in Fig. 7.

In the two previous cases we explored the transferability of the EHT parameters, cf. Table I, optimized for bulk silicon by applying them to other environments such as reconstructed surfaces for silicon (100)- $(2 \times 1)$ and $(111)-(2 \times 1)$. Without any reparametrization the experimentally observed

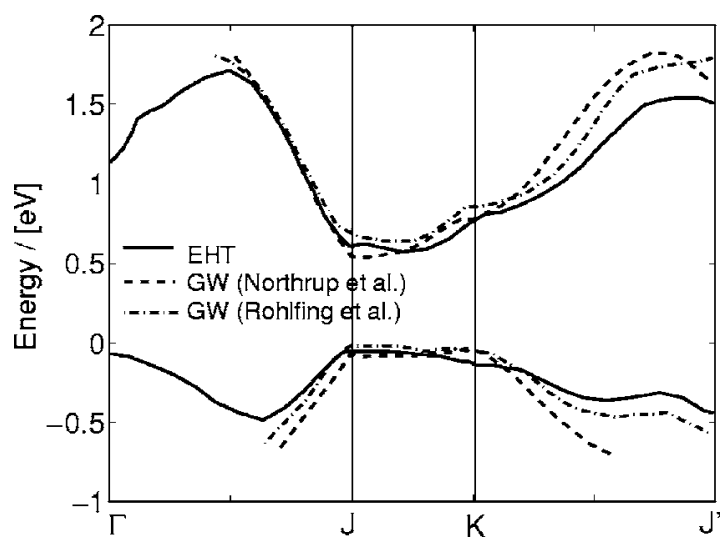

FIG. 7. Comparison of the $\pi$ and $\pi^{*}$ surface bands calculated within EHT and DFT-GW (see Refs. 74 and 75) of Fig. 6 (bottom) (see Ref. 74) and of Fig. 2 from Ref. 75. have been digitized. The data in the dashed curve are adapted with permission from Fig. 2 of Ref. 75. Copyright 1999 American Physical Society. The data in the dashed-dotted curve are adapted with permission from Fig. 2 of Ref. 74. $\pi$ and $\pi^{*}$ surface bands are reproduced quantitatively over a large part of the Brillouin zone, and in the case of silicon (111)- $(2 \times 1)$ over the entire 2D Brillouin zone, as compared to PES/IPES experiments and DFT-GW calculations. As discussed, quantitative differences exist, particularly for the indirect band gap $\Delta_{\pi^{*}-\pi}$ for silicon (100)-( $\left.2 \times 1\right)$, which is underestimated similar to DFT-LDA pseudopotential calculations. $^{73}$

One reason for the quantitative differences, particularly the wrong position of the $\pi$ state in the $\Gamma$-point region which is well above the valence band, might be due to the non-selfconsistent calculation of the band structures for the reconstructed silicon (100) and (111) surfaces. Calculating the total non-self-consistent charge on each dimer atom by integrating the local DOS (LDOS) (effects of the finite sized slab on the LDOS not included) yields a charge of $4.13 e$ on the upper and $3.75 e$ on the lower dimer atom. The total charge of the two dimer atoms is about 7.88e. In turn, a self-consistent calculation of the dimer atom charge using SIESTA (Refs. 76 and 77) results in a total charge on the upper dimer atom of about $4.0 e$, whereas the lower one has $3.88 e$. What one would expect qualitatively is that under selfconsistency the upper atom ( $\pi$ band) which initially carries too much negative charge loses some parts of it to the silicon slab and partial charge is transferred to the lower dimer atom; the net effect is that the $\pi$ band floats down. In turn, the lower dimer atom ( $\pi^{*}$ band) would gain charge partially from the upper dimer atom and from the bulk like slab, so that the respective $\pi^{*}$ band floats up. Since both bands float in opposite directions, the indirect band gap $\Delta_{\pi^{*}-\pi}$ would effectively increase. We expect that a full 3D self-consistent solution of the electronic structure, for example, within a complete neglect of differential overlap (CNDO) scheme, can correct for the discrepancies, which would further increase the transferability of our EHT parameters for silicon.

\section{Si nanowire $\langle 100\rangle: \mathrm{H}$ passivation in EHT}

As a final example, we use the silicon parameters (cf., Table I) to calculate the 1D band structure of a nanowire with square cross section as shown in Fig. 8. The side length of the wire is $L=1.5 \mathrm{~nm}$ and its axis is along the $\langle 100\rangle$ direction. We assume for simplicity that the wire is unrelaxed, and consider (i) an unpassivated surface and (ii) a wire where we have explicitly added hydrogen atoms to saturate the dangling bonds, cf., Fig. 8.

Figure 9 shows the 1D dispersion relation for the two structures. As can be seen in the top part, the bulk band gap of the silicon nanowire is filled with dangling bond states due to the unsaturated bonds of the surface atoms. All dangling bands are completely removed after the wire is passivated by explicitly attaching hydrogen (bottom), so that the original direct band gap of $\sim 2.1 \mathrm{eV}$ is recovered. The nanowire band structure also allows us to calculate transport properties, such as the electronic transmission function (right parts in Fig. 9 at top and bottom). The transmission shows integer step values corresponding to conduction through each wire subband. 

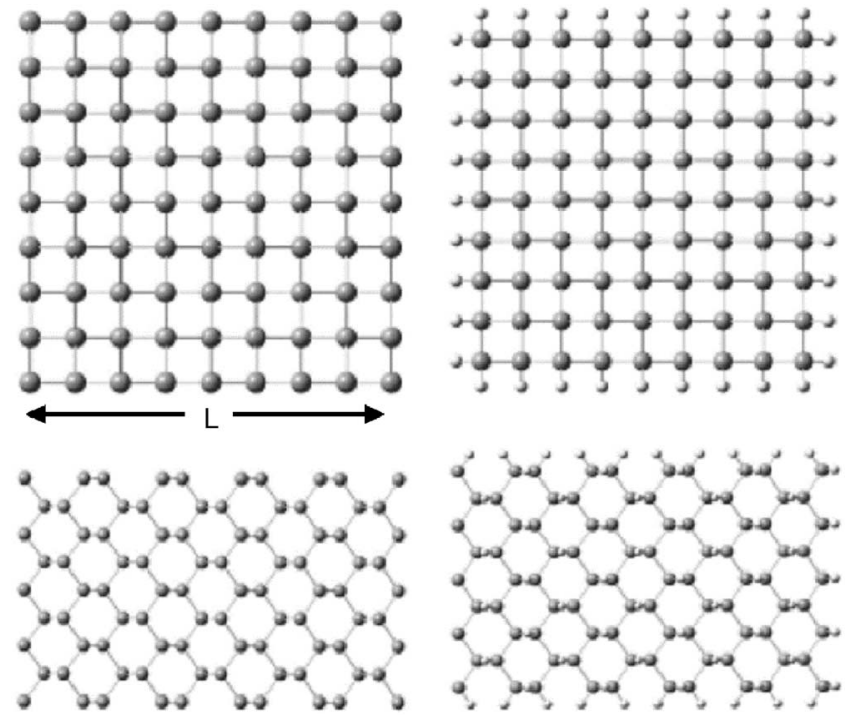

FIG. 8. Front (top) and sideview (bottom) of a silicon nanowire with square cross section and side length $L=1.5 \mathrm{~nm}$ along the $\langle 100\rangle$ direction. For the unpassivated wire (left) the unit cell contains 81 silicon atoms, and in the $\mathrm{H}$-passivated case (right) the total number of atoms is 117.

The extended Hückel scheme allows to passivate surfaces by physically adding surface atoms or molecules to them as shown experimentally for cyclopentene on silicon (100), for example. ${ }^{78}$ This is an improvement over more ad hoc approaches, as in the aforementioned orthogonal tightbinding scheme Ref. 79, for example. In the latter case, the dangling bonds are removed by first transforming the Hamiltonian to a hybrid basis, and then artificially increasing the orbital energies of the relevant unsaturated dangling bonds. While this approach does remove the dangling bond states as desired, the increments in dangling bond onsite energies need to be determined empirically. ${ }^{79}$ Furthermore, the ab-
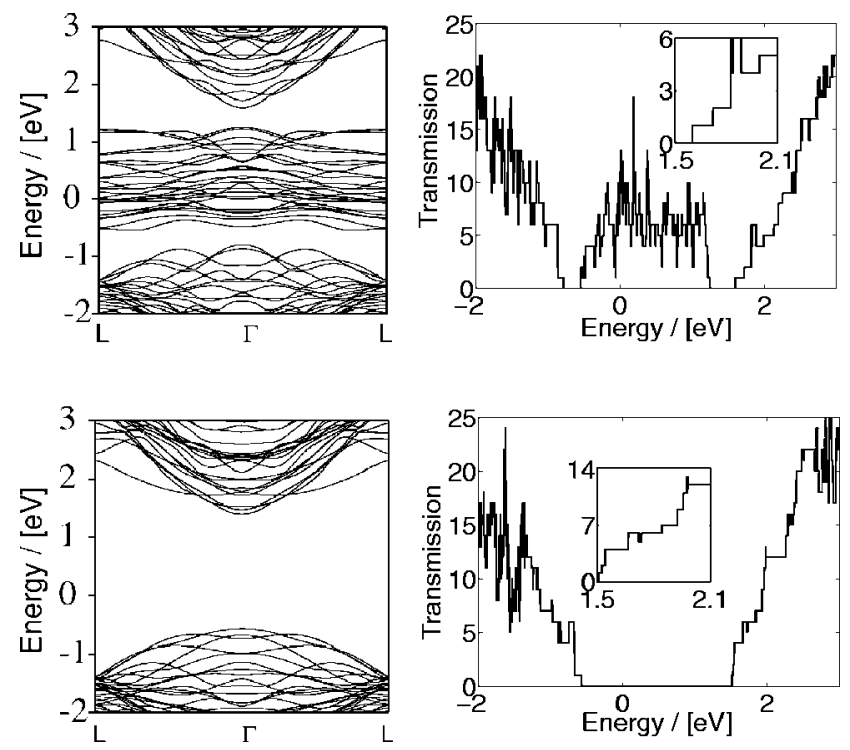

FIG. 9. 1D band structure and transmission per spin of an unrelaxed silicon nanowire along the $\langle 100\rangle$ wire axis. The $E-k$ and transmission at the top are for the unpassivated wire surface, and at the bottom for the H-passivated surface to remove the dangling bond states. The transmission shown in the insets takes integer values where each channel contributes one unit quantum conductance $G_{0}=e^{2} / h$ per spin. sence of actual hydrogen atoms prevents the nanowire wave functions from penetrating out of the well and imposes hardwall boundary conditions that tend to push the charges too far inside from the surface.

In contrast, in extended Hückel theory the surface passivation is controlled by the passivation atoms, which are an explicit part of the entire structure. Once the structure is specified, the amount by which the silicon levels are shifted is determined by the chemical species of the passivation atoms. The hybridization causing the shift of the silicon dangling bond states is naturally incorporated within the Hamiltonian matrix through the EHT-prescription described by Eq. (1). Thus the EHT approach allows us to investigate the role of surface relaxation in nanowire transistors with large surface-to-volume ratios, ${ }^{54}$ and the importance of surface chemistry for nanowire sensors.

\section{SUMMARY}

In summary, we believe that a non orthogonal extended Hückel theoretical (EHT) tight-binding approach (and analogous formulations ${ }^{80,81}$ ) accomplishes a good practical compromise between rigorous, but computationally expensive DFT-based approaches, and orthogonal tight-binding methods, which might not be suitable for large structural deformations beyond $2 \%-3 \%$. The main appeal of EHT is that it captures bulk as well as surface physics along with bonding chemistry at heterointerfaces including large structural deformations, all within a unified semiempirical framework. ${ }^{16,53,59,60}$ The technique still needs further improvement to fully utilize its capabilities and to establish it as a methodological tool towards a quantitative modeling of quantum transport through nanostructures. However, the flexibility of EHT demonstrated here and in our previous paper (Part I $)^{53}$ opens the door to studying the electronic structure and transport through molecular heterostructures as well as larger nanostructures, whose detailed interactions at an atomic scale could nonetheless prove to be quite significant for the understanding and even design of future nanoscale devices.

\section{ACKNOWLEDGMENTS}

The authors acknowledge the support of the Army Research Office through the Defense University Research Initiative in Nanotechnology (DURINT) program, the Defense Advanced Research Projects Agency-Air Force Office of Scientific Research (DARPA-AFOSR), and the Network for Computational Nanotechnology (NCN). One of the authors (J. C.) acknowledges support from the Spanish DGICyT under Contract No. MAT2004-05348-C04-2. The authors are indebted to Tim Boykin, G. Klimeck, and S. Srivastava for helpful discussions.

${ }^{1}$ D. J. Paul, Semicond. Sci. Technol. 19, R75 (2004).

${ }^{2}$ M. Leong, B. Doris, J. Kedzierski, K. Rim, and M. Yang, Science 306, 2057 (2004).

${ }^{3}$ S. Takagi, T. Mizuno, T. Tezuka, N. Sugiyama, S. Nakaharai, T. Numata, J. Koga, and K. Uchida, Solid-State Electron. 49, 684 (2005).

${ }^{4}$ T. Mano, R. Nötzel, D. Zhou, G. J. Hamhuis, T. J. Eijkemans, and J. H. Wolter, J. Appl. Phys. 97, 014304 (2005).

${ }^{5}$ S. A. Healy and M. A. Green, Sol. Energy Mater. Sol. Cells 28, 273 
(1992).

${ }^{6}$ P. Wickboldt, D. W. Pang, W. Paul, J. H. Chen, F. Zhong, C. C. Chen, J. D. Cohen, and D. L. Williamson, J. Appl. Phys. 81, 6252 (1997).

G. Z. Ran, Y. H. Xu, G. L. Ma, A. G. Xu, Y. P. Qiao, W. X. Chen, and G. G. Qin, Semicond. Sci. Technol. 20, 761 (2005).

${ }^{8}$ A. Sazonov, D. Striakhilev, C. H. Lee, and A. Nathan, Proc. IEEE 93, $1420(2005)$

${ }^{9}$ A. G. Xu et al., Phys. Status Solidi A 203, 428 (2006).

${ }^{10}$ J. Collett and D. Villaume, Appl. Phys. Lett. 73, 2681 (1998).

${ }^{11}$ M. Rittner, M. S. Martin-Gonzales, A. Flores, H. Schweizer, F. Effenberger, and M. H. Pilkuhn, J. Appl. Phys. 98, 245306 (2005)

${ }^{12}$ P. G. Piva, G. A. DiLabio, J. L. Pitters, J. Zikovsky, M. Rezeq, S. Dogel, W. A. Hofer, and R. A. Wolkow, Nature (London) 435, 658 (2005).

${ }^{13}$ P. Zhang et al., Nature (London) 439, 703 (2006).

${ }^{14}$ Y. Cui, Q. Wei, H. Park, and C. M. Lieber, Science 293, 1289 (2001).

${ }^{15}$ J. Hahm and C. M. Lieber, Nano Lett. 4, 51 (2004).

${ }^{16}$ G. C. Liang and A. W. Ghosh, Phys. Rev. Lett. 95, 076403 (2005).

${ }^{17}$ N. P. Guisinger, M. E. Greene, R. Basu, A. S. Baluch, and M. C. Hersam, Nano Lett. 4, 55 (2004).

${ }^{18}$ T. Rakshit, G.-C. Liang, A. W. Ghosh, and S. Datta, Nano Lett. 41803 (2004).

${ }^{19}$ T. Rakshit, G.-C. Liang, A. W. Ghosh, M. C. Hersam, and S. Datta, Phys. Rev. B 72, 125305 (2005).

${ }^{20}$ J. L. Pitters and R. A. Wolkow, Nano Lett. 6, 390 (2006).

${ }^{21}$ R. G. Parr and W. Yang, Density-Functional Theory of Atoms and Molecules (Cambridge University Press, New York, 1994).

${ }^{22}$ U. von Barth, Phys. Scr., T T109, 9 (2004).

${ }^{23}$ R. M. Martin, Electronic Structure: Basic Theory and Practical Methods (Cambridge University Press, New York, 1994).

${ }^{24}$ J. R. Reimers, Z. Cai, A. Bili, and N. S. Hush, Ann. N.Y. Acad. Sci. 1006, 235 (2003).

${ }^{25}$ C. Toher, A. Filippetti, S. Sanvito, and K. Burke, Phys. Rev. Lett. 95, 146402 (2005).

${ }^{26}$ W. Lu, V. Meunier, and J. Bernholc, Phys. Rev. Lett. 95, 206805 (2005).

${ }^{27}$ M. S. Hybertsen and S. G. Louie, Phys. Rev. Lett. 55, 1418 (1985).

${ }^{28}$ M. Rohlfing, P. Krüger, and J. Pollmann, Phys. Rev. B 48, 17791 (1993).

${ }^{29}$ P. Vogl, H. P. Hjalmarson, and J. D. Dow, J. Phys. Chem. Solids 44, 365 (1983).

${ }^{30}$ J.-M. Jancu, R. Scholz, F. Beltram, and F. Bassani, Phys. Rev. B 57, 6493 (1998).

${ }^{31}$ T. B. Boykin, G. Klimeck, and F. Oyafuso, Phys. Rev. B 69, 115201 (2004).

${ }^{32}$ L. Perdigao, D. Deresmes, B. Grandidier, M. Dubois, C. Delerue, G. Allan, and D. Stievenard, Phys. Rev. Lett. 92, 216101 (2004).

${ }^{33}$ M. Dubois, L. Perdigao, C. Delerue, G. Allan, B. Grandidier, D. Deresmes, and D. Stievenard, Phys. Rev. B 71, 165322 (2005).

${ }^{34}$ D. J. Chadi, Phys. Rev. Lett. 41, 1062 (1978).

${ }^{35}$ D. J. Chadi, Phys. Rev. Lett. 43, 43 (1979).

${ }^{36}$ D. J. Chadi, Phys. Rev. B 19, 2074 (1979).

${ }^{37}$ D. J. Chadi, Surf. Sci. 99, 1 (1980).

${ }^{38}$ G. V. Hansson, R. Z. Bachrach, R. S. Bauer, D. J. Chadi, and W. Göpel, Surf. Sci. 99, 13 (1980).

${ }^{39}$ D. J. Chadi, Vacuum 33, 613 (1983).

${ }^{40}$ S. Sawada, Vacuum 41, 612 (1990).

${ }^{41}$ J. H. Wilson, J. D. Todd, and A. P. Sutton, J. Phys.: Condens. Matter 2, 10259 (1990)

${ }^{42}$ D. J. Chadi, Surf. Sci. 299, 311 (1994).

${ }^{43}$ D. Tomanek and M. A. Schlüter, Phys. Rev. Lett. 56, 1055 (1986).
${ }^{44}$ E. Kaxiras, Phys. Rev. Lett. 64, 551 (1990).

${ }^{45}$ M. Menon and K. R. Subbaswamy, Phys. Rev. B 47, 12754 (1993).

${ }^{46}$ P. Ordejon, D. Lebedenko, and M. Menon, Phys. Rev. B 50, 5645 (1994).

${ }^{47}$ T. Frauenheim, F. Weich, T. Köhler, S. Uhlmann, D. Porezag, and G. Seifert, Phys. Rev. B 52, 11492 (1995).

${ }^{48}$ P. D. Godwin, A. K. Roberts, D. C. Yu, K. Dean, and P. Clancy, Comput. Mater. Sci. 21, 135 (2001).

${ }^{49}$ B. K. Panda, S. Mukherjee, and S. N. Behera, Phys. Rev. B 63, 045404 (2001).

${ }^{50}$ S. J. Ma and G. H. Wang, J. Mol. Struct.: THEOCHEM 757, 47 (2005).

${ }^{51}$ Z. M. Khakimov, P. L. Tereshchuk, N. T. Sulaymanov, F. T. Umarova, and M. T. Swihart, Phys. Rev. B 72, 115335 (2005).

${ }^{52}$ S. T. Pantelides and J. Pollmann, J. Vac. Sci. Technol. 16, 1349 (1979).

${ }^{53}$ D. Kienle, J. I. Cerda, and A. W. Ghosh, J. Appl. Phys. 100, 043714 (2006).

${ }^{54}$ G.-C. Liang, D. Kienle, S. K. R. Patil, J. Wang, A. W. Ghosh, and S. V. Khare, IEEE Trans. Nanotechnol. (submitted).

${ }^{55} \mathrm{~A}$. W. Ghosh (private communication).

${ }^{56}$ J. C. Slater and G. F. Koster, Phys. Rev. 94, 1498 (1954).

${ }^{57}$ J. N. Murrell and A. J. Harget, Semi-empirical Self-consistent-field Molecular Orbital Theory of Molecules (Wiley-Interscience, New York, 1972).

${ }^{58}$ J. Cerda and F. Soria, Phys. Rev. B 61, 7965 (2000)

${ }^{59}$ D. Kienle and A. W. Ghosh, J. Comput. Electron. 4, 97 (2005).

${ }^{60}$ H. Raza, K. H. Bevan, and D. Kienle, eprint cond-mat/0607226.

${ }^{61}$ C. M. Goringe, D. R. Bowler, and E. Hernandez, Rep. Prog. Phys. 60, 1447 (1997).

${ }^{62}$ A. Pecchia and A. Di Carlo, Rep. Prog. Phys. 67, 1497 (2004).

${ }^{63}$ A. Di Carlo, Semicond. Sci. Technol. 18, R1 (2003).

${ }^{64} \mathrm{~W}$. A. Harrison, Electronic Structure and the Properties of Solids (Freeman, San Francisco, 1980).

${ }^{65}$ P. N. Keating, Phys. Rev. 145, 637 (1966).

${ }^{66}$ The EHT parameters for bulk silicon have been optimized to experimental target values taken from Semiconductors: Group IV Elements and III-V Compounds, edited by O. Madelung (Springer, New York, 1991).

${ }^{67}$ www.icmm.csic.es/jcerda/index.html

${ }^{68}$ H. Takayama, K. P. Bohnen, and P. Fulde, Phys. Rev. B 14, 2287 (1976).

${ }^{69}$ A. Ramstad, G. Brocks, and P. J. Kelly, Phys. Rev. B 51, 14504 (1995).

${ }^{70}$ G. Xu, B. Deng, Z. Yu, S. Y. Tong, A. A. Van Hove, F. Jona, and I. Zasada, Phys. Rev. B 70, 045307 (2004).

${ }^{71}$ M. Rohlfing, P. Krüger, and J. Pollmann, Phys. Rev. B 52, 1905 (1995).

${ }^{72}$ M. Weinelt, M. Kutschera, R. Schmidt, C. Orth, T. Fauster, and M. Rohlfing, Appl. Phys. A: Mater. Sci. Process. 80, 995 (2005). and references therein.

${ }^{73}$ G. P. Srivastava, Theoretical Modelling of Semiconductor Surfaces (World Scientific, Singapore, 1999).

${ }^{74}$ M. Rohlfing and S. G. Louie, Phys. Status Solidi A 175, 17 (1999).

${ }^{75}$ J. E. Northrup, M. S. Hybertsen, and S. G. Louie, Phys. Rev. Lett. 66, 500 (1991).

${ }^{76}$ P. Ordejon, E. Artacho, and J. M. Soler, Phys. Rev. B 53, R10441 (1996).

${ }^{77}$ J. M. Soler, E. Artacho, J. D. Gale, A. Garcia, J. Junquera, P. Ordejon, and D. Sanchez-Portal, J. Phys.: Condens. Matter 14, 2745 (2002).

${ }^{78}$ R. Akiyama, T. Matsumoto, and T. Kawai, Phys. Rev. B 62, 2034 (2000).

${ }^{79}$ S. Lee, F. Oyafuso, P. von Allmen, and G. Klimeck, Phys. Rev. B 69, 045316 (2004).

${ }^{80}$ T. Frauenheim, G. Seifert, M. Elstner, Z. Hajnal, G. Jungnickel, D. Porezag, S. Suhai, and R. Scholz, Phys. Status Solidi A 217, 41 (2000).

${ }^{81}$ T. Frauenheim et al., J. Phys.: Condens. Matter 14, 3015 (2002). 